\title{
Multiple- and single-objective approaches to laminate optimization with genetic algorithms
}

\author{
L. Costa, L. Fernandes, I. Figueiredo, J. Júdice, R. Leal, P. Oliveira
}

\begin{abstract}
In this paper an application of a genetic algorithm to a material- and sizing-optimization problem of a plate is described. This approach has obvious advantages: it does not require any derivative information and it does not impose any restriction, in terms of convexity, on the solution space. The plate optimization problem is firstly formulated as a constrained mixed-integer programming problem with a single objective function. An alternative multiobjective formulation of the problem in which some constraints are included as additional objectives is also presented. Some numerical results are included that show the appropriateness of the algorithm and of the mathematical model for the solution of this optimization problem, as well as the superiority of the multiobjective approach.
\end{abstract}

Key words genetic algorithms, multiple objective optimization, combinatorial optimization

\section{1}

\section{Introduction}

Sizing or material structural optimization problems are, in general, formulated as nonlinear continuous problems

Received: 12 July 2002

Revised manuscript received: 28 May 2003

Published online: 30 January 2004

(C) Springer-Verlag 2004

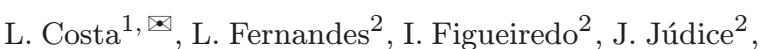
R. Leal $^{3}$, P. Oliveira ${ }^{1}$

${ }^{1}$ Departamento de Produção e Sistemas, Escola de Engenharia, Universidade do Minho, Campus de Gualtar, 4710 Braga, Portugal

e-mail: lac@dps .uminho.pt; pno@dps .uminho.pt

2 Departamento de Matemática, Universidade de Coimbra, Apartado 3008, 3001-454 Coimbra, Portugal

e-mail: Imerca@co.it.pt; Isabel.Figueiredo@mat.uc.pt; Joaquim. Judice@co.it.pt

${ }^{3}$ Departamento de Engenharia Mecânica, Universidade de Coimbra, Pinhal de Marrocos, 3030-201 Coimbra, Portugal e-mail: rogerio.leal@dem.uc.pt
(Haslinger and Neittaanmäki 1998; Bendsøe and Soares 1993; Bendsøe 1995). The numerical solutions are obtained by conventional gradient algorithms that require derivative as well as convexity properties and that usually converge to local optimal solutions. However, modeling real-world problems imposes (by both natural and technological reasons) the consideration of integer variables, such as different materials and/or different thickness, chosen among a set of integer values. This leads to mixed-integer nonlinear problems, lacking the properties of differentiability and convexity. Deb and Goyal (1998), in the context of mechanical component design, refer to the problems that can emerge when using classical optimization techniques, which are designed to use continuous variables, in dealing with integer variables at the expense of introducing additional constraints.

Evolutionary algorithms (Goldberg 1989), in contrast to other descent algorithms, do not require any differentiability or convexity conditions and seem to be appropriate for the solution of this type of problem. Moreover, since these algorithms start from a pool of points, they are less prone to being trapped in a local optimum and consequently find a global minimum or at least a good solution to the problem. Several approaches have used genetic algorithms in structural topology optimization (Hajela et al. 1992; Kane and Schoenauer 1996). Other reported approaches to composite laminated optimization using genetic algorithms have also been published (Le Riche and Haftka 1993; Le Riche et al. 1999; Costa et al. 1999), as well as approaches based on multiobjective optimization (Hajela and Lin 1992; Grosset et al. 2001). The structural optimization problem considered here is concerned with the stiffness of a linearly laminated elastic plate for which the optimization variables are the thickness and the material of each lamina. This leads to a combinatorial problem since different orders of the materials define different stiffness of the plate. The material variables assume discrete values, with thickness assuming values in a continuous set. Furthermore, constraints on this problem can also be considered, such as upper bounds on the mass, price, and global thickness of the plate.

The structural model under consideration can be formulated as an optimization problem with a single objective, the compliance of the structure, and some con- 
straints associated with the thickness, price, and mass involved in the structure (Fernandes et al. 1998; Costa et al. 2000). The use of genetic algorithms to process this optimization problem imposes these constraints to become part of an exact penalty function. It then seems natural to consider instead a multiobjective formulation (Deb 2001) of the structural model, with four objective functions associated with the compliance, thickness, price, and mass of the structure. Numerical experience reported in this paper seems to indicate that this latter multiobjective approach (Costa et al. 2001) is more appropriate for processing the structural model.

The organization of this paper is as follows. In Sect. 2, the general formulation of the plate optimization problem as a constrained mixed-integer programming problem is described, together with the single- and multipleobjective formulations. The application of genetic algorithms to process this problem is discussed in Sect. 3. The multiobjective genetic algorithm is introduced in Sect. 4 . Computational experience with the genetic algorithm is reported in Sect. 5, with some conclusions and a few hints for future research in Sect. 6.

\section{2}

\section{Formulation of the problem}

\section{1}

\section{Single objective formulation}

We consider a thin elastic plate, made of several laminas which are symmetric with respect to the middle plane of the plate defined by the set $\Omega \subset R^{2}$. Due to this symmetry, the total number of layers composing the plate is equal to $2 k$, where $k$ is an integer greater than 1 . Each lamina, with thickness $t_{i}$, is associated exactly with one material $m_{j}$.

We define the vectors

$t=\left(t_{1}, \ldots, t_{k}\right) \quad$ and $\quad m=\left(m_{1}, \ldots, m_{p}\right)$,

which correspond to the vectors of thickness and materials, respectively.

The structural optimization problem consists of finding the best material and thickness distribution that yields the stiffest plate. The discrete formulation of this model, which is obtained by the finite element method, corresponds to the following minimization problem:

$$
\left[\begin{array}{l}
\min \frac{1}{2} f^{T} u \\
(t, m) \in C \\
\text { subject to } K(t, m) u=f .
\end{array}\right.
$$

The variables appearing in problem (2), that is, the optimization variables, the vectors $u$ and $f$, the matrix $K$, and the constraint set $C$, are discussed below. a) The vectors $t \in R^{k}$ and $m \in R^{p}$ contain the optimization variables $t_{i}$ and $m_{j}$ associated with the thickness and materials of the plate, respectively.

To define the thickness $t_{i}$ of each lamina $i=1,2, \ldots, k$, let $h_{i}$ be the distance from the middle surface $\Omega$ to the upper face of the lamina $i$. So $t_{i}=h_{i}-h_{i-1}$ is the thickness of lamina $i$ and

$2 \sum_{i=1}^{k} t_{i}=2 \sum_{i=1}^{k}\left(h_{i}-h_{i-1}\right)$

is the total thickness of the plate $\left(h_{0}=0\right)$.

Each material $m_{j}$, with $j=1,2, \ldots, p$, of the admissible set of materials, is defined by a set of elastic properties depending on the material considered. As each lamina $i$ must have a unique material $m_{j}$, chosen among a set of $p$ materials, the material $l_{i}$ in lamina $i$ is given by

$l_{i}=\sum_{j=1}^{p} x_{i j} m_{j}, \quad$ for $\quad i \in\{1,2, \ldots, k\}$,

where

$\sum_{j=1}^{p} x_{i j}=1, \quad$ for $\quad i=1, \ldots, k$,

and

$x_{i j} \in\{0,1\}, \quad$ for $\quad i=1, \ldots, k, \quad j=1, \ldots, p$.

If repeated materials are not allowed the additional constraint

$\sum_{i=1}^{k} x_{i j}=1, \quad$ for $\quad j=1, \ldots, p$,

is also considered. This condition (6) enables a better understanding of the mechanical results. In what follows, we denote by $x$ the $k \times p$ matrix with components $\left(x_{i j}\right)$.

b) The vector $u \in R^{q}$ represents the approximation of the vertical displacement of the plate, at given points of the middle surface ( $q$ is the number of global degrees of freedom of the finite element mesh).

c) The vector $f \in R^{q}$ is the vertical force that acts on the plate.

d) The square matrix $K(t, m)$, where $m$ is related to $x$ by (3), in what follows denoted by $K(t, x)$, is the global stiffness matrix (positive semi-definite matrix of order $q$ ) obtained from the element stiffness matrices following standard displacement finite element procedures. At the element level $(e)$ the stiffness matrix may be written as

$K^{(e)}(t, x)=\int_{\Omega^{(e)}} B^{(e)^{T}} D^{(e)}(t, x) B^{(e)}$,

where

$D^{(e)}(t, x)=\sum_{i=1}^{k} D_{i}^{(e)}\left(t_{i}, l_{i}\right)$. 
The definitions of the elasticity matrix $D^{(e)}(t, x)$ and the strain displacement matrix $B^{(e)}=L S^{(e)}$, where $L$ is a matrix of differential operators and $S^{(e)}$ is the shape function matrix, are dependent on the choice of the material of each lamina, the laminate theory, and the finite element.

e) The set $C$ is the set of admissible thickness and materials, and it includes some constraints, such as upper and lower bounds on the thickness, as well as an upper bound for the global thickness, price, and mass of the plate. Next we introduce these constraints. As far as the thickness vector $t$ is concerned, the constraints are defined by the expressions

$$
\left[\begin{array}{l}
t_{i}^{\min } \leq t_{i} \leq t_{i}^{\max }, \quad i=1, \ldots, k \\
t_{i}^{\min } \text { and } t_{i}^{\max } \text { known for each } i \\
\sum_{i=1}^{k} t_{i}^{\min } \leq \sum_{i=1}^{k} t_{i} \leq T_{1}<\sum_{i=1}^{k} t_{i}^{\max }
\end{array}\right.
$$

where $T_{1}$ is a constraint on the total thickness. Furthermore, the total mass should be smaller or equal to a given constant $M_{1}$. This can be written as

$S \sum_{i=1}^{k} t_{i} \sum_{j=1}^{p} x_{i j} \rho_{j} \leq M_{1}$

where $S$ is the area of the middle surface of the plate, $\rho_{j}$ is the density of mass of the material $j$ and $x_{i j}$ are defined in (3)-(5). Finally, the total price must be smaller than a fixed price $P_{1}$, whence

$S \sum_{i=1}^{k} t_{i} \sum_{j=1}^{p} x_{i j} \rho_{j} p_{j} \leq P_{1}$,

where $p_{j}$ is the relative price of material $j$, and $S$ and $x_{i j}$ are the same as in (10).

It then follows that the plate optimization problem can be formulated as the following mixed-integer program

$$
\begin{array}{ll}
\text { Minimize } & g_{1}(t, x)=\frac{1}{2} f^{T} u \\
\text { Subject to } & K(t, x) u=f \\
& t_{i}^{\min } \leq t_{i} \leq t_{i}^{\max }, \quad i=1, \ldots, k \\
& \sum_{j=1}^{p} x_{i j}=1, \quad i=1, \ldots, k \\
& \sum_{i=1}^{k} x_{i j}=1, \quad j=1, \ldots, p, \\
& x_{i j} \in\{0,1\}, \quad i=1, \ldots, k, \quad j=1, \ldots, p \\
& g_{2}(t, x) \leq T_{1}, \\
& g_{3}(t, x) \leq M_{1} \\
& g_{4}(t, x) \leq P_{1}
\end{array}
$$

where $t=\left(t_{i}\right) \in R^{k}, x=\left(x_{i j}\right) \in R^{k \times p}, K(t, x)$ is the $q \times q$ global stiffness matrix defined before, $f \in R^{q}$, and $g_{2}, g_{3}$, and $g_{4}$ are functions defined as follows:

$g_{2}(t, x)=\sum_{i=1}^{k} t_{i}$,

$g_{3}(t, x)=S \sum_{i=1}^{k} t_{i} \sum_{j=1}^{p} x_{i j} \rho_{j}$,

$g_{4}(t, x)=S \sum_{i=1}^{k} t_{i} \sum_{j=1}^{p} x_{i j} \rho_{j} p_{j}$.

In this mathematical program, the constraints (14)(17) should be distinguished, as they are easily satisfied by fixing a unique variable $x_{i j}$ to 1 , all the remaining variables to zero, and forcing each one of the variables $t_{i}$ to belong to the interval with the corresponding lower and upper bounds, $t_{i}^{\min }$ and $t_{i}^{\max }$, respectively. Furthermore, the value of the objective function for such a solution is easy to compute by solving the linear system (13) after previously fixing the values of the variables $t$ and $x$ according to $(7)$.

\section{2}

\section{Multiple-objective formulation}

The plate optimization problem can naturally be formulated as a multiobjective problem. To this end, the difficult constraints (18)-(20) are now considered as objectives, thus defining a multiobjective model with four objective functions $g_{r}$, for $r=1,2,3,4$, subject to the set of the easy constraints (14)-(17), as well as to the system (13). Therefore, the multiobjective formulation of the plate problem is the following:

$$
\begin{array}{ll}
\text { Minimize } & \left\{g_{r}(t, x), \quad r=1,2,3,4\right\} \\
\text { subject to } & K(t, x) u=f, \\
& t_{i}^{\min } \leq t_{i} \leq t_{i}^{\max }, \quad i=1, \ldots, k \\
& \sum_{j=1}^{p} x_{i j}=1, \quad i=1, \ldots, k, \\
& \sum_{i=1}^{k} x_{i j}=1, \quad j=1, \ldots, p, \\
& x_{i j} \in\{0,1\}, \quad i=1, \ldots, k, \quad j=1, \ldots, p .
\end{array}
$$

In this case, the search space does not have a complete order, which requires a different notion of optimality. This notion demands the definition of Pareto dominance. For any two decision vectors $\left(t^{1}, x^{1}\right)$ and $\left(t^{2}, x^{2}\right)$,

i) if $g_{r}\left(t^{1}, x^{1}\right) \leq g_{r}\left(t^{2}, x^{2}\right)$ for all $r$, and $g_{r}\left(t^{1}, x^{1}\right)<$ $g_{r}\left(t^{2}, x^{2}\right)$ for at least one $r$, then $\left(t^{1}, x^{1}\right)$ dominates $\left(t^{2}, x^{2}\right)$, represented by $\mathbf{g}\left(t^{1}, x^{1}\right) \prec \mathbf{g}\left(t^{2}, x^{2}\right)$; 
ii) if $\mathbf{g}\left(t^{1}, x^{1}\right) \nprec \mathbf{g}\left(t^{2}, x^{2}\right)$ and $\mathbf{g}\left(t^{2}, x^{2}\right) \nprec \mathbf{g}\left(t^{1}, x^{1}\right)$, then $\left(t^{1}, x^{1}\right)$ is indifferent to $\left(t^{2}, x^{2}\right)$;

iii) a point $\left(t^{1}, x^{1}\right)$ is non-dominated in a given subset of the feasible region if there is no other solution in that set that dominates it, i.e., $\nexists\left(t^{2}, x^{2}\right): \mathbf{g}\left(t^{2}, x^{2}\right) \prec \mathbf{g}\left(t^{1}, x^{1}\right)$. A point $\left(t^{1}, x^{1}\right)$ is said to be a Pareto optimal point if and only if it is non-dominated in the entire feasible set.

For the case of just two objectives $g_{1}$ and $g_{2}$, Fig. 1 shows the dominance relations between four solutions $a$, $b, c$, and $d$. Dark gray areas signal regions of space dominated by each solution. Similarly, with respect to each solution, light gray areas depict regions of space that might contain solutions that dominate it. Thus, solution $a$ dominates solutions $c$ and $d$ and is indifferent to $b$; solution $b$ dominates solution $d$ and is indifferent to $a$ and $c$; solution $c$ dominates solution $d$ and is dominated by solution $a$ and indifferent to $b$; solution $d$ is dominated by solutions $a, b$, and $c$.

Unlike the single-objective formulation, the multiobjective model produces a set of solutions expressing the different compromises that can be reached when considering the different objectives (the trade-offs between cost, mass, thickness, and compliance). The set of nondominated solutions is referred as the Pareto optimal set.

There are some approaches in which a weighted sum of the objectives is considered (Hajela and Lin 1992; Grosset et al. 2001), which permits the multiple-objective problem to be addressed as a single objective. However, for non-convex Pareto fronts this approach may not find all the solutions (Zitzler et al. 2000; Deb 2001). Furthermore, in order to have a definition of the Pareto front, several sets of weights must be considered, thus demanding several runs of the algorithm.

Another possible approach is to use the $\varepsilon$-constraint method (Miettinen 1999), in which one of the objectives is selected to be optimized and the other objectives are converted into constraints by setting an upper bound to

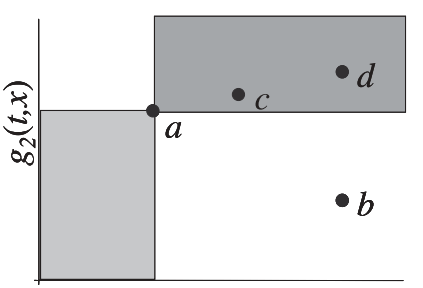

$g_{1}(t, x)$

Solution $a$

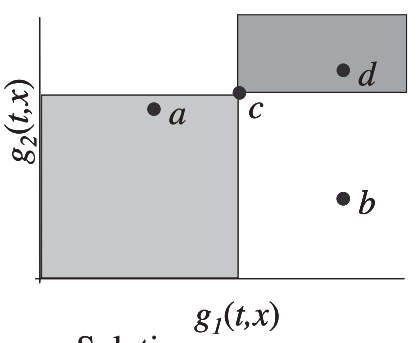

Solution $c$

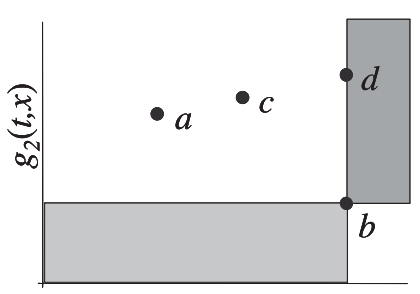

Solution $b$

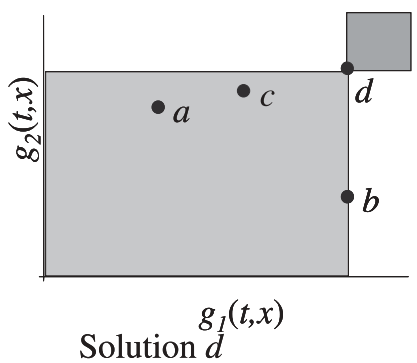

Solution $d$
Fig. 1 Dominance relations each of them. This approach avoids the problem of nonconvex fronts, but the setting of the upper bounds may be difficult, requiring for each objective several executions.

To understand the connection between single and multiobjective formulations, notice that if $(\bar{t}, \bar{x})$ is a Pareto optimal point for the four objective functions $g_{r}, r=1,2,3,4$, then $(\bar{t}, \bar{x})$ is a global minimum for the single-objective optimization problem, where

$T_{1}=g_{2}(\bar{t}, \bar{x}), M_{1}=g_{3}(\bar{t}, \bar{x}), P_{1}=g_{4}(\bar{t}, \bar{x})$.

This easily follows from the definitions of a global minimum and a Pareto optimal solution (Miettinen 1999).

An algorithm solely based on the values of the objective function seems to be recommended to process the single-objective mixed-integer program, provided it incorporates a penalty technique to deal with the difficult constraints (18)-(20). Moreover, the algorithm can be modified in order to solve the multiobjectve mixedinteger program. In the next sections a genetic algorithm is introduced that uses a penalty or a multiobjective approach for such a purpose.

\section{A single-objective genetic algorithm}

\section{1 \\ Genetic coding - chromosome representation}

The implementation of a genetic algorithm for the material- and sizing-optimization problem of a plate requires the consideration of a two-level approach: the structural problem solution (which is the solution of the equation $K(t, x) u=f)$ and the minimization problem (12)-(20). For a given set of materials, together with the respective thickness, the solution of the structural problem can be achieved through the finite element program. Each set of materials $l_{i}$ and thickness $t_{i}$, can be seen as a point in the search space for the genetic algorithm. Thus, the structural solution is the performance measure associated with each chromosome, a set of materials and thickness. Each chromosome is evaluated through the finite element program, thus allowing a ranking of every chromosome present in the population at a given generation.

In the first instance, a rectangular symmetric plate is considered with a maximum number of ten layers, and with no repeated materials. Therefore, due to symmetry, only five layers are required to be considered, that is, $k=5$. Thus, a plate is described by five continuous variables, denoting the thickness of each layer; these variables are coded using a binary representation of 4 bits, thus leading to a set of 20 bits. Since each layer is made of only one material, and there are no repeated materials, five integer ordinal variables were used to represent the order of the materials in the plate. It is possible to have a layer with zero thickness, which corresponds to a plate with only eight layers. However, a solution with all laminas of 
Example for $k=2 \times 5$ and $p=9$

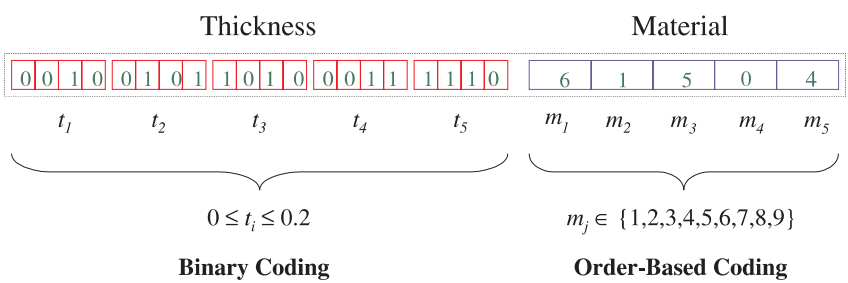

Fig. 2 Chromosome coding

zero thickness is not allowed. Thus each chromosome has a total length of 25 positions (alleles), where 20 alleles represent the continuous variables and 5 the integer ordinal variables (Fig. 2).

\section{2}

\section{Genetic operators}

The implementation of the various genetic operators does not create any special difficulties, since each one is applied independently to the two types of variables in the chromosome representation. For each kind of variable (continuous $t_{i}$ or integer $m_{j}$ ), different genetic operators are developed. For the continuous variables $t_{i}$, a twopoint crossover and uniform mutation are implemented with different probabilities. An ordinal crossover (uniform order-based) with a mask and a scramble sublist mutation are implemented for the integer ordinal vari-

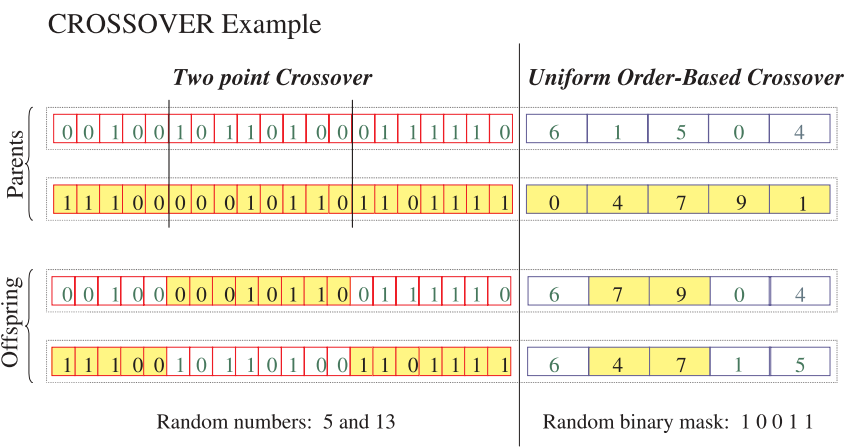

Fig. 3 Crossover

\section{MUTATION Example}

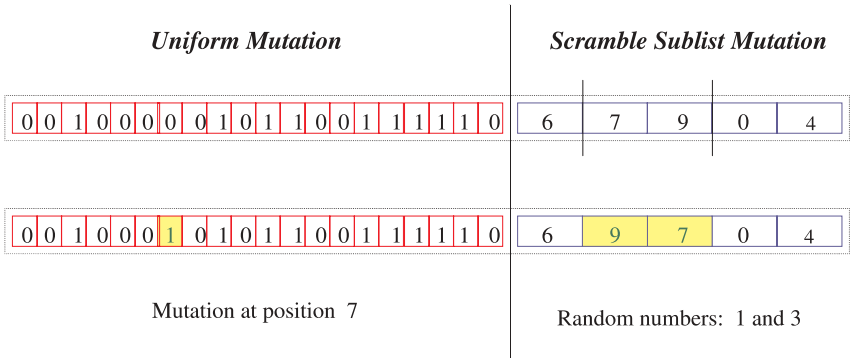

Fig. 4 Mutation ables (Figs. 3 and 4). Like the two-point crossover for binary strings, the ordinal crossover preserves part of the first parent and incorporates information from the second parent, in spite of its implementation being a little more complex. Next, the process of generating the first offspring from the two parents (for the second offspring the complemental process is carried out) is presented:

1. a random binary mask is created;

2 . if, at a given position, the bit mask is 1 then the gene is copied from the first parent;

3. the remaining positions are filled with the genes of the second parent that are different from the genes copied previously from the first parent (according to the order they appear).

The scramble sublist mutation selects randomly a sublist of the parent, and then the order of the elements of the sublist is reversed. These genetic operators were developed to prevent the generation of illegal solutions.

\section{3 \\ Constraints and objective function}

Constraint handling in evolutionary algorithms has mostly been addressed by penalization techniques (Goldberg 1989; Deb 2000; Michalewicz et al. 1996). Michalewicz et al. (1996) provide an overview of constraint handling, referring to the rejection and repair of unfeasible individuals, the use of decoders, special representations, and genetic operators, the penalization of unfeasible solutions, and the approach to constrained optimization as multiobjective optimization methods to incorporate constraint violations.

Several constraints were considered as indicated in (14)-(20), namely the maximum and minimum thickness of each layer (14), the total thickness of the plate (18), the total mass of the plate (19), and the total price of the plate (20).

In our approach, the genetic operators were chosen so as to prevent the generation of unfeasible solutions with respect to the constraints (14)-(16). The difficult constraints (18)-(20) that restrict the search space are included in the objective function through a penalty term, leading to the following function:

$L(t, x, u)=\frac{1}{2} f^{T} u+R \sum_{i=1}^{3}\left[C_{i}(t, x)\right]^{2}$,

where $R$ is the penalty coefficient and the functions $C_{i}(t, x), i=1,2,3$, are defined by

$\left[\begin{array}{l}C_{1}(t, x)=\min \left\{0, T_{1}-g_{2}(t, x)\right\}, \\ C_{2}(t, x)=\min \left\{0, M_{1}-g_{3}(t, x)\right\}, \\ C_{3}(t, x)=\min \left\{0, P_{1}-g_{4}(t, x)\right\},\end{array}\right.$

with $g_{i}$ the functions given by (21)-(23). 
3.4

\section{Outline of the genetic algorithm}

Figure 5 shows the outline of the genetic algorithm. Firstly, a random population of solutions is generated, each one evaluated through the finite element code. Selection of the best solutions takes place by tournament selection (a solution is selected based on the comparison with a pre-specified set of solutions, the tournament set (Goldberg 1989)), after which the application of the genetic operators gives rise to a new population. The procedure is applied until the solutions converge or a pre-specified number of generations is reached. The GA parameters are fixed as in Table 2

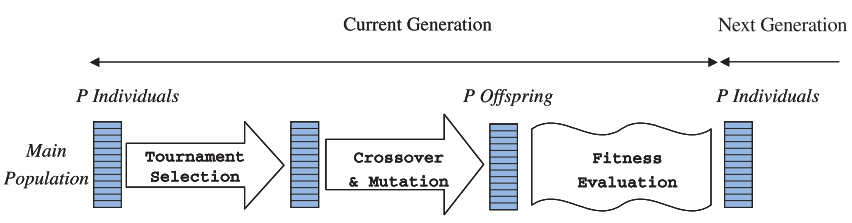

Fig. 5 Outline of a genetic algorithm

\section{4}

\section{A multiobjective genetic algorithm}

Many algorithms exist for multiobjective optimization with genetic algorithms (Deb 2001). In this work, we use an approach (Costa et al. 2001) based on the NonDominated Sorting Genetic Algorithm (NSGA) by Srinivas and Deb (1994), in which the aim is to approximate the Pareto front. The main differences with a singleobjective GA lie in fitness evaluation, for which the concept of domination has to be introduced; moreover, in order to achieve a balanced distribution of the solutions along the Pareto front, a sharing mechanism (Goldberg 1989) must be introduced by which the fitness of each solution is degraded in accordance with the number of solutions that lie in its neighborhood (Euclidean distance). Thus, convergence to part of the Pareto front is prevented.

In each generation non-dominance is tested in order to guide the algorithm in the direction of the Pareto set. At each generation, the non-dominated solutions $n_{1}$ in the gene pool are defined as the first front. It should be noted that these solutions are indifferent to each other. Thus, these solutions have a performance measure equal to $P$, the size of the population. In order to preserve diversity, a sharing mechanism is implemented, degrading the assigned performance of neighboring solutions, in the hypersphere with radius defined by the parameter sigma share. Next, these solutions are temporarily ignored, and the set of non-dominated points in the remaining solutions constitutes the second front. The performance measure attributed to these solutions is equal to the minimum assigned performance in the previous front minus 1 . Thus, any solution in front $k$ will have a performance measure below any solution in front $k-$ 1. Sharing is again applied to the solutions in this front. Sharing can either be implemented on the genotypic distance (the encoding distance) or in the phenotypic distance (either the decoded decision variables distance or the objective function values distance). In what concerns phenotypic distance, several arguments exist that lead to one approach (decision variable distance) being preferred to the other (objective function distance). The likely existence of multiple solutions on the decision space with the same objective function value might favor the first approach if it is considered that diversity on the decision space is of primary importance; however, the Pareto set is defined on the objective function values and, thus, it might be of greater importance to have diversity, i.e., a well-balanced distribution on the objective function space. We solve the problem considering sharing on the objective domain (phenotypic distance). Further details of the algorithm can be found in Costa et al. (2001).

\section{1}

\section{An elitist multiobjective genetic algorithm}

In spite of the success of the application of multiobjective genetic algorithms, some authors (Zitzler et al. 2000) suggest that elitism can speed up its performance and also prevent the loss of good solutions found during the search process. Thus, the previous algorithm incorporates an elitist technique based on a separate secondary population (SP) that contains all (or a part of) potential Pareto optimal solutions found so far during the search process. In this sense, SP is completely independent of the main population, as all its solutions do not participate in the search process. A specified number, $\theta$, of these elite solutions (indifferent Pareto solutions) is introduced in the main population.

In its simplest form, the new potential Pareto optimal solutions that are found are stored in the SP, in all generations. The SP update implies the determination of Pareto optimality of all solutions stored so far, in order to eliminate those that become dominated. As the size of the SP grows, the time to complete this operation may become significant. In order to prevent this increase, a maximum size for the SP is imposed. For all generations, a potential Pareto optimal solution $x_{n d}$ found in the main population is stored if

1. all solutions in the SP are different from $x_{n d}$

2. none of the solutions in the SP dominates $x_{n d}$.

In order to control the size of the SP, a new parameter $d>0$ is introduced that represents the minimum desirable Euclidean distance on the objective space between potential Pareto optimal solutions in the SP. The previous Step 2 of the algorithm is modified as follows:

2. none of the solutions in the SP dominates $x_{n d}$ and the distance from $x_{n d}$ to any of the solutions in the SP which are not dominated by $x_{n d}$ is greater than $d$. 
This elitist strategy (Costa et al. 2001) has several advantages when compared with the results solely from the main population:

- the set of all non-dominated solutions in the SP constitutes a far better approach to the optimal Pareto set;

- the solutions in the SP clearly present a balanced distribution along the Pareto front;

- parameter $d$ allows the definition of the concentration of points all along the Pareto front;

- the size of the SP is small when compared with other approaches;

- the additional computational time required is negligible, considering the quality of the results when compared with just one main population; moreover, the computational time and effort is much lower than that required to maintain all non-dominated points in the SP.

It should be added that the bookkeeping of the nondominated solutions in the SP requires efficient algorithms in order to reduce the computational effort. Figure 6 presents a schematic representation of the algorithm.

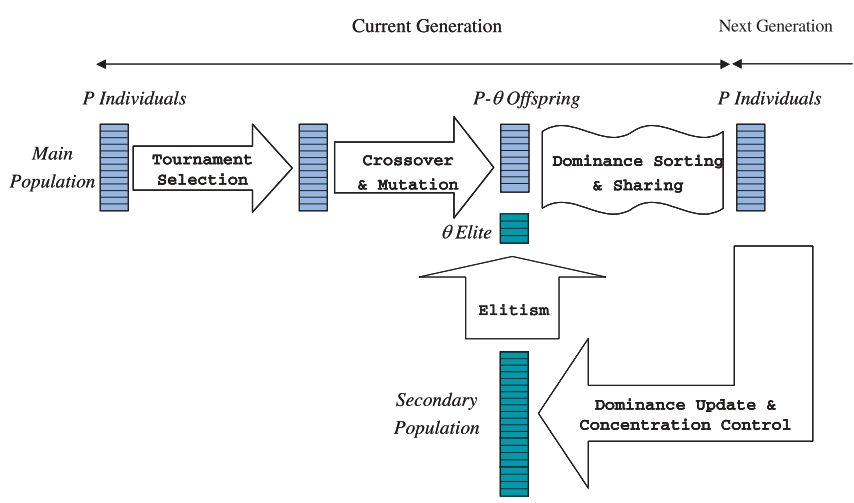

Fig. 6 Outline of the multiobjective elitist genetic algorithm

\section{5}

\section{Numerical results}

The main objectives of our experiences are to verify if the solutions obtained by the genetic algorithms have physical meaning and to compare the single-objective and the multiobjective approaches discussed in this paper. In order to have a problem instance which allows a clear and simple interpretation of the results, the list of materials considered in Table 1 was chosen accordingly. Furthermore, we define the problem as a mixed-integer programming problem and we impose some artificial constraints by fixing the number of layers, by imposing maximum limits for the layer thickness, mass, and price. Some additional constraints are also introduced in order to avoid repeated materials in the plate.

Each material is characterized by a Young's modulus, a Poisson's ratio, a specific mass, and a relative price. For a given chromosome, specifying a set of materials with the respective thickness, the CALFEM package (10) is used to evaluate the solution $u$ of the equation $K(t, x) u=f$. The Kirchhoff plate finite element "platre" of the code CALFEM (LTH 1997, cf.p.5.7-1) is used to compute the stiffness matrix $K_{i}$ of each lamina $i$. This finite element "plate" is a rectangle, suited for a linear elastic isotropic material, with twelve degrees of freedom (the four displacements and the two derivatives of the vertical displacement, at the vertices of the rectangle). The solution $u$ is then incorporated together with the thickness, mass, and price constraints (18), (19), and (20), in the objective function defined by (31).

It should be noted that the problem that we have constructed to be processed by the algorithms is not really a real-world structural optimization problem because, with a good choice of materials, an experienced engineer can decide the optimum laminate configuration in almost every case, without the use of any optimization algorithm. It is obvious that in a plate subjected to bending loads, the maximum stresses are on the faces while the minimum stresses are on the middle plane. Thus, the materials should be arranged in increasing order of their stiffness starting from the middle plane. Moreover, the set of materials listed in Table 1 is not applicable in a realworld structural optimization problem. In fact, copper, bronze, glass, lead, and rubber exhibit poor mechanical characteristics and are expensive when compared with steel (the reference material), and nickel is much more expensive than steel for almost similar mechanical properties.

If we consider a real-world combinatorial optimization problem like a similar problem with reinforced epoxy with carbon or glass with variable material, discrete thickness, and discrete angle in each lamina, the results will be much more difficult to analyze (Costa et al. 2001).

Several preliminary experiments were carried out in order to tune the genetic algorithm parameters. These experiments included the variation of the crossover and mutation probabilities. The crossover probability was chosen among the values $0.6,0.7$, and 0.8 , and the mutation probability from $0.0005,0.001$, and 0.005 . After the tuning phase, the parameters were fixed as in Table 2.

The runs were executed on a personal computer with a Pentium III processor. It should be noted that $95 \%$ of the total execution time was devoted to the execution of the subroutine CALFEM that finds the displacement vector $u$ (mostly due to the interface with MATLAB). Thus, for the problems considered in our experiments, the objective function evaluation had a high computational cost. On average, each function evaluation took 1.6 seconds. Furthermore, for the single-objective formulation, during the search process, the same solutions are often evaluated, especially when the population is about to converge. Thus, a scheme that minimizes the repetition of the same calculations seems to be very useful for this kind of problem. The implemented scheme maintained a list containing the objective function values more of- 
Table 1 List of materials

\begin{tabular}{lrlrl}
\hline Material & $\begin{array}{l}\text { Young's } \\
\text { Modulus } \\
\left(\mathrm{kg} / \mathrm{cm}^{2}\right)\end{array}$ & $\begin{array}{l}\text { Poisson's } \\
\text { Ratio }\end{array}$ & $\begin{array}{l}\text { Specific } \\
\text { Mass } \\
\left(\mathrm{kg} / \mathrm{cm}^{3}\right)\end{array}$ & $\begin{array}{l}\text { Price } \\
(\text { Relative } \\
\text { Price } / \mathrm{kg})\end{array}$ \\
\hline \hline Steel (S) & $21 \times 10^{5}$ & 0.28 & $7.8 \times 10^{-3}$ & 1 \\
Nickel (N) & $20.7 \times 10^{5}$ & 0.29 & $7.75 \times 10^{-3}$ & 6.25 \\
Iron (I) & $20 \times 10^{5}$ & 0.28 & $7.4 \times 10^{-3}$ & 0.65 \\
Copper (C) & $11 \times 10^{5}$ & 0.34 & $8.9 \times 10^{-3}$ & 6.25 \\
Bronze (B) & $10 \times 10^{5}$ & 0.31 & $8.25 \times 10^{-3}$ & 4.5 \\
Aluminium (A) & $7 \times 10^{5}$ & 0.34 & $2.6 \times 10^{-3}$ & 5 \\
Glass (G) & $5.5 \times 10^{5}$ & 0.25 & $2.7 \times 10^{-3}$ & 3.75 \\
Lead (L) & $1.8 \times 10^{5}$ & 0.44 & $11.34 \times 10^{-3}$ & 3 \\
Rubber (R) & $0.037 \times 10^{5}$ & 0.485 & $1.8 \times 10^{-3}$ & 5 \\
\hline
\end{tabular}

Table 2 The GA parameters

\begin{tabular}{lcc}
\hline Parameter & Single objective & Multiobjective \\
\hline \hline Number of experiments & 4 & 1 \\
$\begin{array}{l}\text { Maximum number of } \\
\text { generations }\end{array}$ & 1000 & 250 \\
$\begin{array}{l}\text { Population size } \\
\text { Two-point crossover }\end{array}$ & 100 & 200 \\
$\begin{array}{l}\text { probability } \\
\text { Uniform mutation } \\
\text { probability }\end{array}$ & 0.7 & 0.7 \\
$\begin{array}{l}\text { Uniform order-based } \\
\text { crossover probability }\end{array}$ & 0.001 & 0.01 \\
$\begin{array}{l}\text { Scramble sublist mutation } \\
\text { probability }\end{array}$ & 0.001 & 0.7 \\
$\begin{array}{l}\text { Penalty coefficient } \\
\text { Sigma share }\end{array}$ & 1000 & 0.1 \\
$\begin{array}{l}\text { Minimal distance between } \\
\text { potential solutions }\end{array}$ & - & - \\
\hline
\end{tabular}

ten used along the successive generations. This scheme allowed the reduction, on average, of $15 \%$ of the total objective function evaluations (the execution time was also reduced proportionally).
Table 3 Problem instances

\begin{tabular}{lllll}
\hline Problem & $\begin{array}{l}\text { Layer's } \\
\text { Thickness } \\
(\mathrm{mm})\end{array}$ & $\begin{array}{l}\text { Total } \\
\text { Thickness } \\
(\mathrm{mm})\end{array}$ & $\begin{array}{l}\text { Total } \\
\text { Mass } \\
(\mathrm{kg})\end{array}$ & $\begin{array}{l}\text { Total } \\
\text { Relative } \\
\text { Price }\end{array}$ \\
\hline \hline$\# 0$ & $\leq 0.2$ & - & - & - \\
$\# 1$ & $\leq 0.2$ & $\leq 0.8$ & $\leq 2$ & $\leq 10$ \\
$\# 2$ & $\leq 0.2$ & $\leq 0.8$ & $\leq 2$ & $\leq 5$ \\
$\# 3$ & $\leq 0.2$ & $\leq 0.8$ & $\leq 1$ & $\leq 5$ \\
$\# 4$ & $\leq 0.2$ & $\leq 0.5$ & $\leq 2$ & $\leq 5$ \\
$\# 5$ & $\leq 0.2$ & $\leq 0.8$ & $\leq 0.8$ & $\leq 5$ \\
$\# 6$ & $\leq 0.2$ & $\leq 0.8$ & $\leq 2$ & $\leq 1$ \\
\hline
\end{tabular}

Several experiments (listed in Table 3) were performed with the single-objective formulation in order to test whether the model could produce results with physical meaning. For every experiment, a restriction on the maximum thickness of each layer $(0.2 \mathrm{~mm})$ was considered. Problem \#0 depicts the experiment with no restrictions except on the thickness of each layer. Problems \#1 to \#6 present the conjunction of different restrictions on the total mass, total thickness, and relative price.

Table 4 presents the best solutions obtained, with the list of materials from the outer to the inner layer, together with the respective thickness. In this table, \#Gen and \#Eval are the mean number of generations and objective function evaluations observed, respectively.

Table 4 Best solutions

\begin{tabular}{llllllllllllll}
\hline Probl. & \multicolumn{4}{c}{ Materials } & \multicolumn{4}{c}{ Layer's Thickness (mm) } & \multicolumn{3}{c}{ \#Gen } & \#Eval \\
\hline \hline$\# 0$ & S & N & I & C & B & 0.20 & 0.20 & 0.20 & 0.20 & 0.20 & 29 & 1693 \\
$\# 1$ & S & N & I & A & G & 0.20 & 0.16 & 0.20 & 0.11 & 0.13 & 70 & 4824 \\
$\# 2$ & N & S & I & A & G & 0.13 & 0.20 & 0.13 & 0.16 & 0.17 & 69 & 4622 \\
$\# 3$ & I & S & A & G & R & 0.08 & 0.07 & 0.19 & 0.20 & 0.20 & 72 & 4787 \\
$\# 4$ & S & N & I & G & R & 0.19 & 0.08 & 0.15 & 0.01 & 0.07 & 70 & 4642 \\
$\# 5$ & S & N & I & A & R & 0.04 & 0.05 & 0.05 & 0.20 & 0.20 & 79 & 4810 \\
$\# 6$ & S & I & & & & 0.20 & 0.19 & 0.00 & 0.00 & 0.00 & 65 & 4415 \\
\hline
\end{tabular}




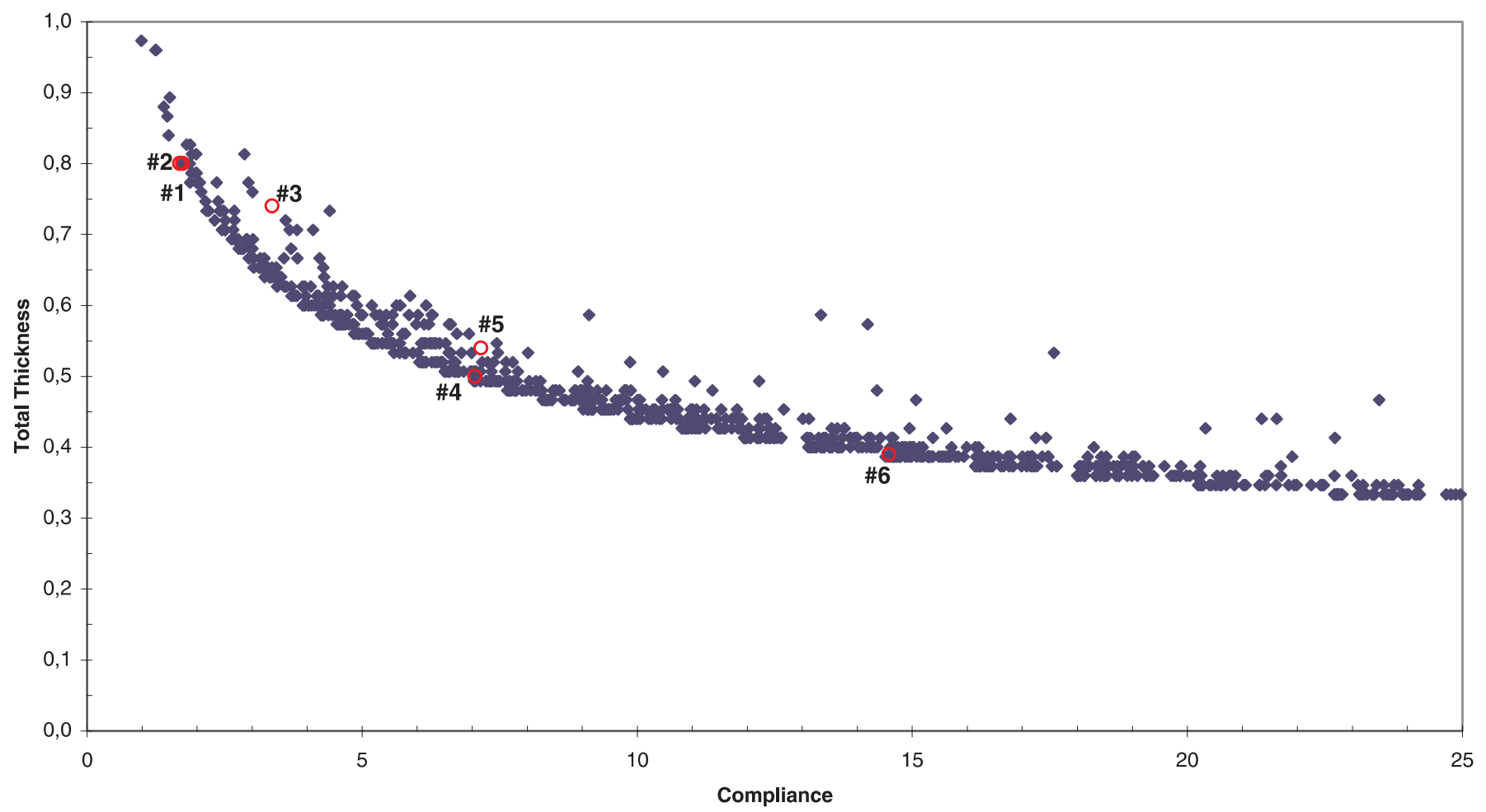

Fig. 7 Non-dominated solutions at 250th generation - total thickness vs compliance (sharing on the variable domain)

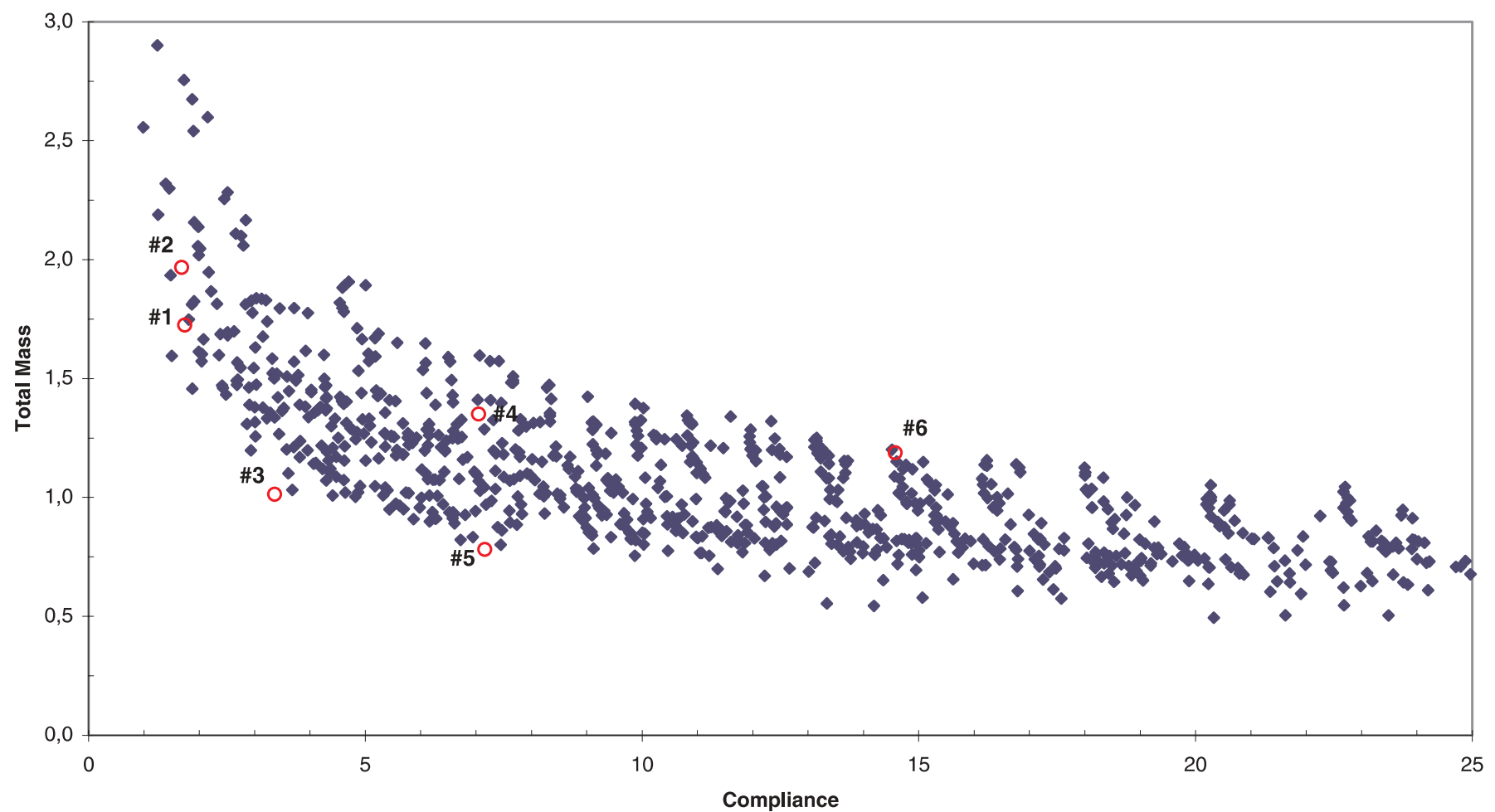

Fig. 8 Non-dominated solutions at 250th generation - total mass vs compliance (sharing on the variable domain)

The results agree with the physical analysis of the problem. Thus, in the absence of constraints (Problem $\# 0$ ) the maximum allowable thickness is obtained and the materials are set in increasing order of stiff- ness from the middle plane to the exterior. On the other hand, in the cases where price is more constrained (Problems \#2 to \#6) the most expensive materials contribution is reduced or eliminated from the stacking, with the 


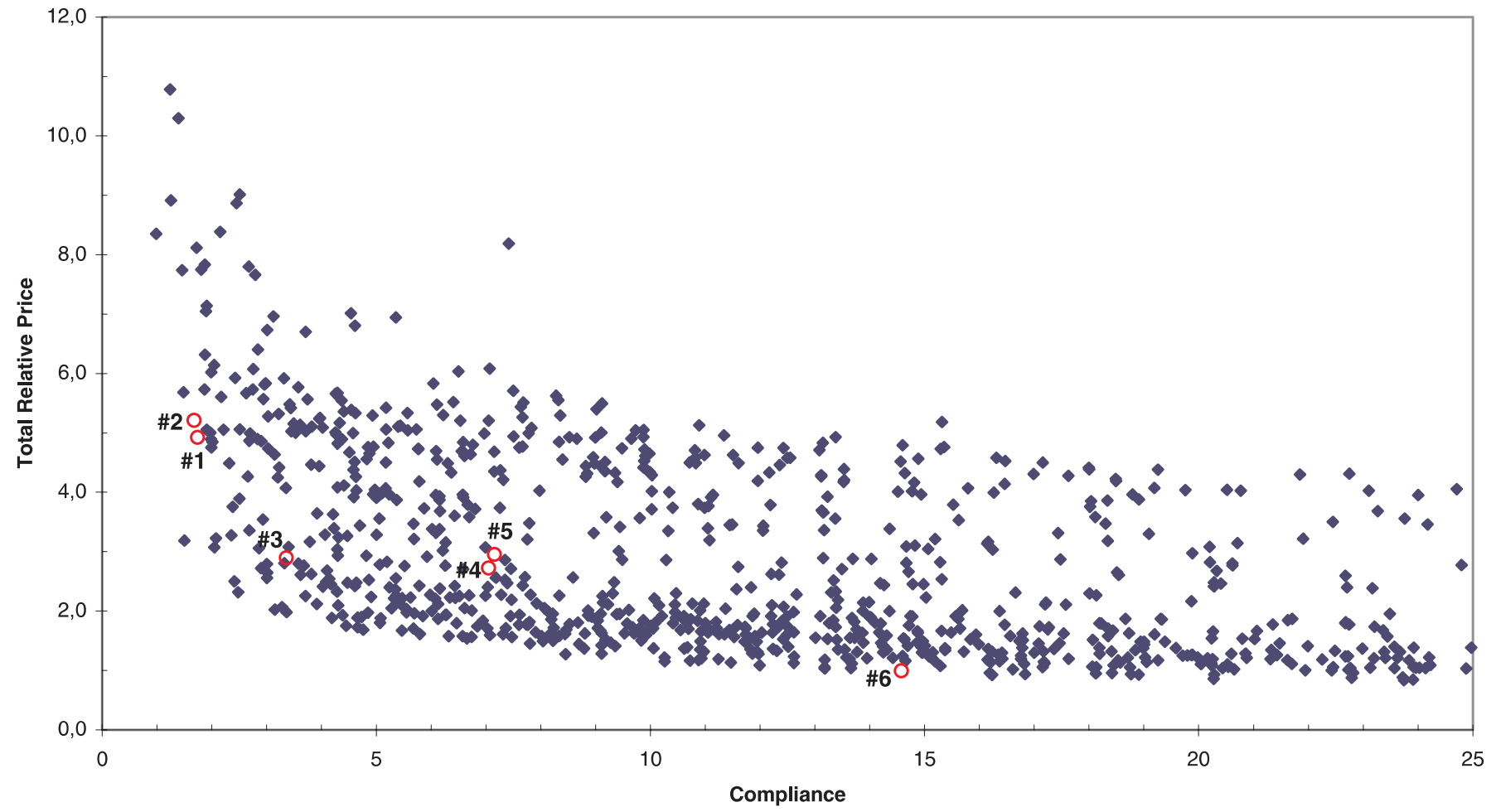

Fig. 9 Non-dominated solutions at 250th generation - total relative price vs compliance (sharing on the variable domain)

remaining materials keeping the increasing order of the mechanical properties; in the limit, when the price is extremely low (Problem \#6), the total thickness of the plate is reduced and the materials which are selected are the ones that exhibit the best relation between mechanical properties and price.

Figures 7, 8, and 9 show the non-dominated solutions in the SP (potential Pareto solutions) at the end of 250 generations with sharing on the variable domain. The $2 \mathrm{D}$ plots are presented as a function of the most important objective (the compliance).

It can be observed that the algorithm produced a welldefined non-dominated front. The single-objective solutions generated from problems \#1 to \#6 are included in the two-dimensional plots of the front. It can be seen that these points are in the non-dominated set, in spite of the fact that some of them are located at extreme values of the feasible set. Moreover, the several projections of the front, together with a look-up table with the nondominated points, permits the decision maker to choose an operating point, defining, for instance, a limit on the compliance which should not be overcome and achieving the compromises corresponding to the other objectives.

\section{6}

\section{Conclusions}

In this paper, a plate optimization problem has been formulated as a mixed-integer nonlinear-programming problem. Due to the lack of differentiability and convexity of the problem, a genetic algorithm has been proposed for its solution, in which the constraints are incorporated in the objective function through a penalty scheme. It has been shown that this problem can also be formulated as a multiobjective problem in which the further objectives are concerned with the restrictions on the mass, thickness, and price of the single-objective optimization.

In the multiobjective formulation, there is no single optimum. A set of points is instead constructed representing trade-offs between the different objectives. This set of points constitutes the Pareto optimal set and any multiobjective algorithm must be able to approximate this set. As genetic algorithms are based on the generation of populations, they seem to be quite suited to approximating the Pareto front.

Different sets of approximations to the Pareto optimal set are generated along the successive generations produced by the genetic algorithm. A scheme has been implemented which keeps a subset of representative nondominated solutions produced by the algorithm as a secondary population. This secondary population is clearly a major contribution for well-defined non-dominated fronts. Numerical results included in this paper show that the proposed implementation is quite recommended for the job, since the increase in the computational effort is compensated by the clear definition of the fronts.

Several single-objective minimizations have been carried out in order to evaluate the approximation to the Pareto set. In these problem instances, the restrictions have been set at extreme values of the feasible set. The difficulties in obtaining a set of non-dominated solutions containing all the generated single-objective optimal solutions has shown the importance of the independent ap- 
plication of the genetic operators to the continuous and ordinal parts of the chromosomes. Moreover, the importance of having high mutation rates on the ordinal variables has also been noticed. In fact, the non-dominated set may not contain the single-objective optimal solutions, in case this process does not come into operation. The use of extreme points of the feasible set ensures that the generated non-dominated fronts are good approximations to the Pareto optimal set.

The evaluation of the Pareto approximation can be visualized by the graphic representation of the solutions. However, the graphic representation may not be clear for more than two objectives. It should be pointed out that all the points on the $2 \mathrm{D}$ plots are non-dominated. The relative position for some points may seem to contradict the previous statement, but this is the result of the projection of a four-dimensional figure on the two-dimensional plots. Moreover, the decision maker may observe the tradeoff that must be considered when choosing a particular point (solution) in the Pareto set. Finally the multiobjective genetic approach is quite interesting in this particular application, as all the efficient solutions of the restricted problem can be obtained in one run. All these considerations lead to our final conclusion that the multiobjective approach is clearly superior to the single-objective one for the structural problem discussed in this paper.

Acknowledgements This work is part of the project "New materials, adaptive systems and their nonlinearities; modelling, control and numerical simulation" carried out in the framework of the european community program "Improving the human research potential and the socio-economic knwledge base" (HRN-CT-2002-00284), and is partially supported by the projects PRAXIS/PCEX/P/MAT/38/96 and FCT-POCTI/34471/MAT/2000 of Portugal.

\section{References}

Bendsøe, M.P. 1995: Optimization of structural topology, shape, and material. Berlin: Springer

Bendsøe, M.P.; Soares, C.M. 1993: Topology design of structures. Dordrecht: Kluwer Academic Press

Costa, L.; Oliveira, P.; Figueiredo, I.; Leal, R. 1999: Compliance minimization of a composite laminated plate by genetic algorithms. In: Proceedings of the European Conference on Computational Mechanics ECCM99 (held in Munich, Germany), pp. 740-741

Costa, L.; Oliveira, P.; Figueiredo, I.; Roseiro, L.; Leal, R. 2000: Structural optimization of laminated plates with genetic algorithms. In: Whitley, D.; Goldberg, D.; Cantu-Paz, E.; Spector, L.; Parmee, I.; Beyes, H.-G. (eds.) Proceedings of the Genetic and Evolutionary Computation Conference GECCO 2000, pp. 621-627. (held in Las Vegas, USA) Morgan Kaufman Publishers

Costa, L.; Oliveira, P.; Figueiredo, I.; Roseiro, L.; Leal, R. 2001: Laminated plate design optimization using a multiobjective genetic algorithm. In: Evolutionary methods for design, optimisation and control with applications to industrial prob- lems - Proceedings of the Eurogen 2001 (held in Athens, Greece), pp. 391-396

Deb, K.; Goyal, M. 1998: A flexible optimization procedure for mechanical component design based on genetic adaptive search. Trans. ASME 120, 162-164

Deb, K. 2000: An efficient constraint handling for genetic algorithms. Comput. Methods Appl. Mech. Eng. 186(2)-(4), $311-338$

Deb, K. 2001: Multi-objective optimization using evolutionary algorithms. Chichester: John Wiley \& Sons

Fernandes, L.; Figueiredo, I.; Júdice, J.; Costa, L.; Oliveira, P. 1998: Application of genetic algorithms to plate optimization. In: Idelsohn, S.R.; Oñate, E.; Dvorkin, E. (eds.) Computational mechanics, new trends and applications. Barcelona: CIMNE

Goldberg, D. 1989: Genetic algorithms in search, optimization, and machine learning. Reading, MA: Addison-Wesley

Grosset, L.; Venkataraman, S.; Haftka, R. 2001: Genetic optimization of two-material composite laminates. ASC Conference (held in Blacksburg)

Hajela, P.; Lin, C.-Y. 1992: Genetic search strategies in multicriterion optimal design. Struct. Optim. 4, 99-107

Hajela, P.; Lee, E.; Lin, C.-Y. 1992: Genetic algorithms in structural topology optimization. In: Bendsøe, M.P.; Soares, C.M. (eds.) Topology design of structures, pp. 117-133. Dordrecht: Kluwer Academic Press

Haslinger, J.; Neittaanmäki, P. 1998: Finite element approximation for optimal shape design, theory and applications. Chichester: John Wiley \& Sons

Kane, C.; Schoenauer, M. 1996: Topological optimum design using genetic algorithms. Control Cybern. 25(5), 1054-1087

Le Riche, R.; Haftka, R. 1993: Optimization of laminated stacking sequence for buckling load maximization by genetic algorithm. AIAA J. 31(5), 951-956

Le Riche, R.; Knopf-Lenoir, C.; Haftka, R. 1995: A segregated genetic algorithm for constrained structural optimization. In: Eshelman, L. (ed.) Proceedings of the Sixth International Conference on Genetic Algorithms, Morgan Kaufmann, San Francisco, CA, pp. 558-565

LTH 1997: Lund University, CALFEM, A finite element toolbox to MATLAB, version 3.2, Sweden

Michalewicz, Z.; Dasgupta, D.; Le Riche, R.; Schoenauer, M. 1996: Evolutionary algorithms for constrained engineering problems. Comput. Ind. Eng. J. 30(2), 851-870

Miettinen, K.M. 1999: Nonlinear multiobjective optimization. Boston: Kluwer Academic Publishers

Reddy, J.N. 1997: Mechanics of laminated composite plates. Theory and Analysis. Boca Raton, FL: CRC Press

Schwefel, H.-P. 1995: Evolution and optimum seeking. New York: John Wiley \& Sons

Srinivas, N.; Deb, K. 1994: Multi-objective function optimization using non-dominated sorting genetic algorithms. Evol. Comput. 2(3), 221-248

Zitzler, E.; Deb, K.; Thiele, L. 2000: Comparison of multiobjective evolutionary algorithms: Empirical results. Evol. Comput. 8, 173-195 